\title{
New successful one-step surgical repair for apple peel atresia
}

\author{
This article was published in the following Dove Press journal: \\ Open Access Surgery \\ 2I November 201। \\ Number of times this article has been viewed
}

\section{Mahmoud Machmouchi \\ Department of Pediatrics, Royal Commission Hospital, Jubail, Saudi Arabia}

Correspondence: Mahmoud Machmouchi Department of Pediatrics, Royal Commission Hospital, PO Box 10402, Jubail 3196I, Saudi Arabia

Tel +96633464000

Fax +9663346I700

Email drmachmouchi@gmail.com
Abstract: A new successful surgical approach in two identical twins delivered with intestinal atresia, "apple peel" type is reported. This technique consists of: (1) an end-to-end oblique primary anastomosis with single layer inverted 5/0 Vicryl ${ }^{\circledR}$ sutures (Ethicon, Inc, Somerville, NJ); (2) proximal tube jejunostomy using Foley catheter, size 10 French, inserted through a stab wound in the left upper quadrant and entering the proximal dilated loop at about $10 \mathrm{~cm}$ proximal from its blind end (site of anastomosis); and (3) transanastomotic stenting using feeding tube, size 6 French, exteriorized in conjunction with the Foley catheter and reaching the lumen of the distal loop for more than $20 \mathrm{~cm}$. The postoperative course was uneventful and progressive oral feed became ad libitum around day 40 postoperative. This procedure is simple, performed in one stage, and responds to the most obligatory requirements of this congenital malformation. Keywords: proximal jejunal atresia, intestinal atresia, surgical repair, anastomosis

\section{Introduction}

Intestinal atresia is a congenital malformation causing neonatal intestinal obstruction. "Apple peel" type is rare but still presents serious surgical repair challenges with high morbidity and mortality. A new successful surgical approach is presented, with the hope that it will be adopted as a first-line therapeutic approach for apple peel atresia.

\section{Case reports}

Two identical twins were delivered at full-term via cesarean section, following an uneventful pregnancy to a healthy 26 -year-old gravida 1 para 1 mother. There was no consanguinity and their birth weights were $1460 \mathrm{~g}$ and $1995 \mathrm{~g}$, respectively.

Diagnosis of neonatal intestinal obstruction was made prenatally by ultrasound at the end of the seventh month. The ultrasound showed an intraabdominal structural abnormality, suspected to be an abnormal dilated intestinal loop. No other abnormality, except for a very mild polyhydramnios, was noticed at the time of the ultrasound, nor during cesarean section. In both of the twins, postnatal assessment revealed the presence of mild systolic murmur, related to a patent ductus arteriosus.

After birth, there was a significant amount of bile-stained gastric aspirate; plain abdominal X-ray confirmed the presence of high intestinal obstruction with a short proximal dilated loop (Figure 1). After postnatal evaluation, informed parental consent was obtained and the babies were operated on during the first 36 hours.

Exploration confirmed a jejunal atresia about $15 \mathrm{~cm}$ from the ligament of Treitz, with complete separation of blind-end loops and extensive mesenteric defect giving 


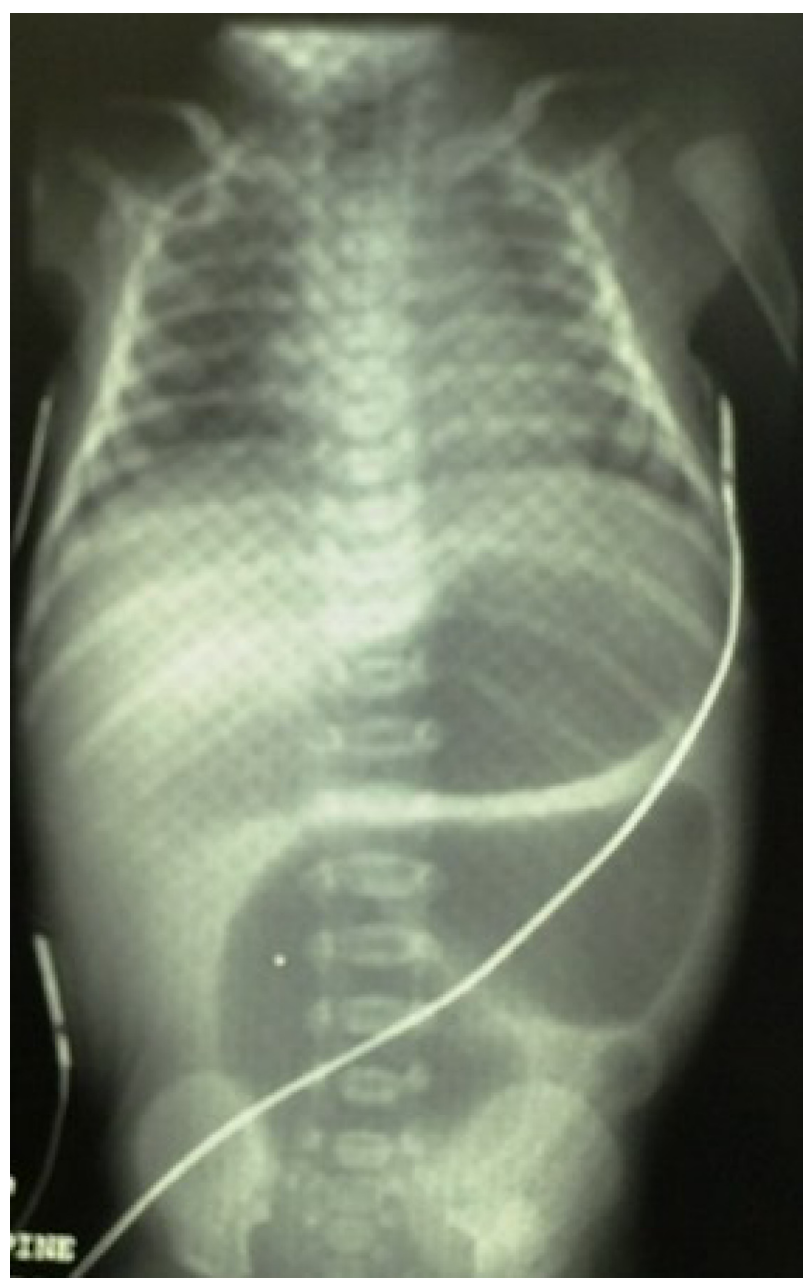

Figure I Abdominal preoperative X-ray, twin I.

the typical appearance of apple peel atresia. The distal small intestine was short (less than $40 \mathrm{~cm}$ ), with coils around the vascular axis. The disparity in lumen diameter between the proximal and distal tiny loops was about 15 folds (Figure 2).

The operation consisted of (Figure 3):

1. An end-to-end oblique anastomosis with a single layer inverted 5/0 Vicryl ${ }^{\circledR}$ sutures (Ethicon, Inc, Somerville, NJ).

2. Proximal tube jejunostomy constructed using Foley catheter (Foley catheter latex-free manssield MA02048; Tyco/Healthcare Kendall, Mexico City, Mexico), size French 10, inserted through a short stab wound in the left upper quadrant and in the proximal dilated loop about $10 \mathrm{~cm}$ proximal to the anastomosis.

3. Transanastomotic stent using a feeding tube (Feeding Tube, Nontoxic Pyrogene Free; Kawamoto Corporation, Tokyo, Japan), size French 6, exteriorized in conjunction with the Foley catheter. The foley catheter and stent

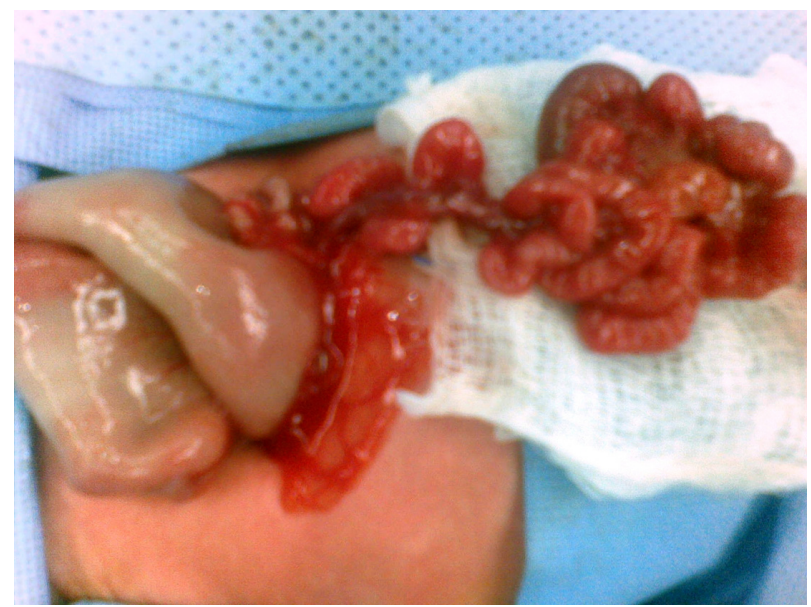

Figure 2 Macroscopic appearance of the intestine during laparotomy, twin I.

were inserted through a jejunal stab wound, closed by 3/0 Vicryl purse-string suture, the balloon was inflated, and the intestine was fixed to the inner abdominal wall by three opposite 3/0 Vicryl sutures. Finally, the Foley catheter and transanastomotic tube were fixed to the skin by a nonabsorbable suture to prevent their dislodgment.

Postoperative course was uneventful. Initially, Foley catheter drained about $80-100 \mathrm{~mL}$ of bile-stained fluid per day; this drainage progressively decreased to $0 \mathrm{~mL}$ by day 15. Abdominal X-ray showed the presence of air in the distal small intestine on day 5 postoperative (Figure 4). Oral feeding started on day 15 with $2 \mathrm{~mL} \mathrm{5 \%}$ dextrose half-normal saline every 8 hours, which was increased gradually and then changed to half strength and later to full strength milk
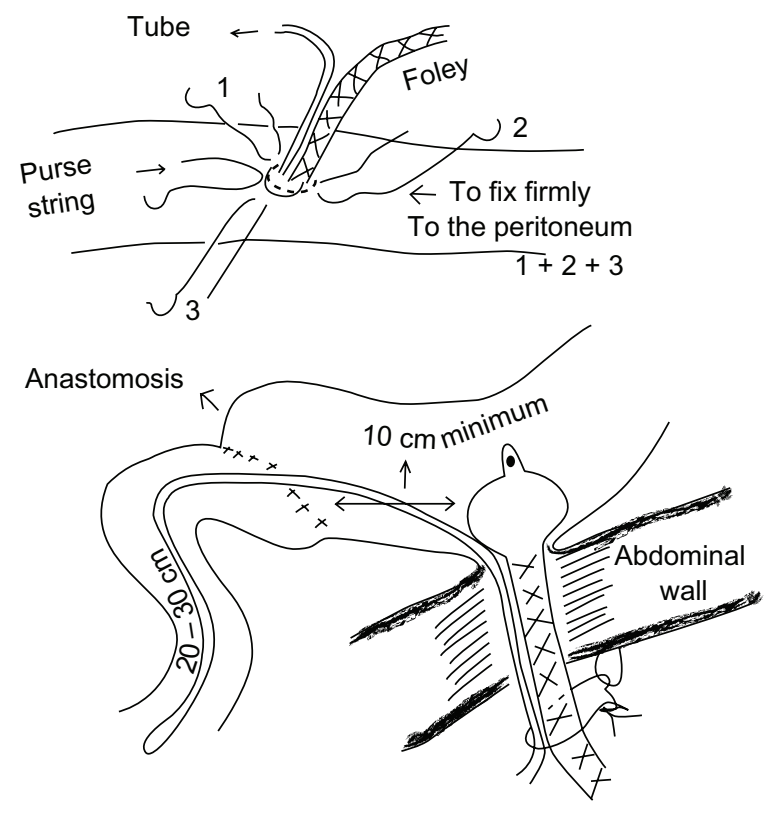

Figure 3 Illustration of the surgical procedure. 


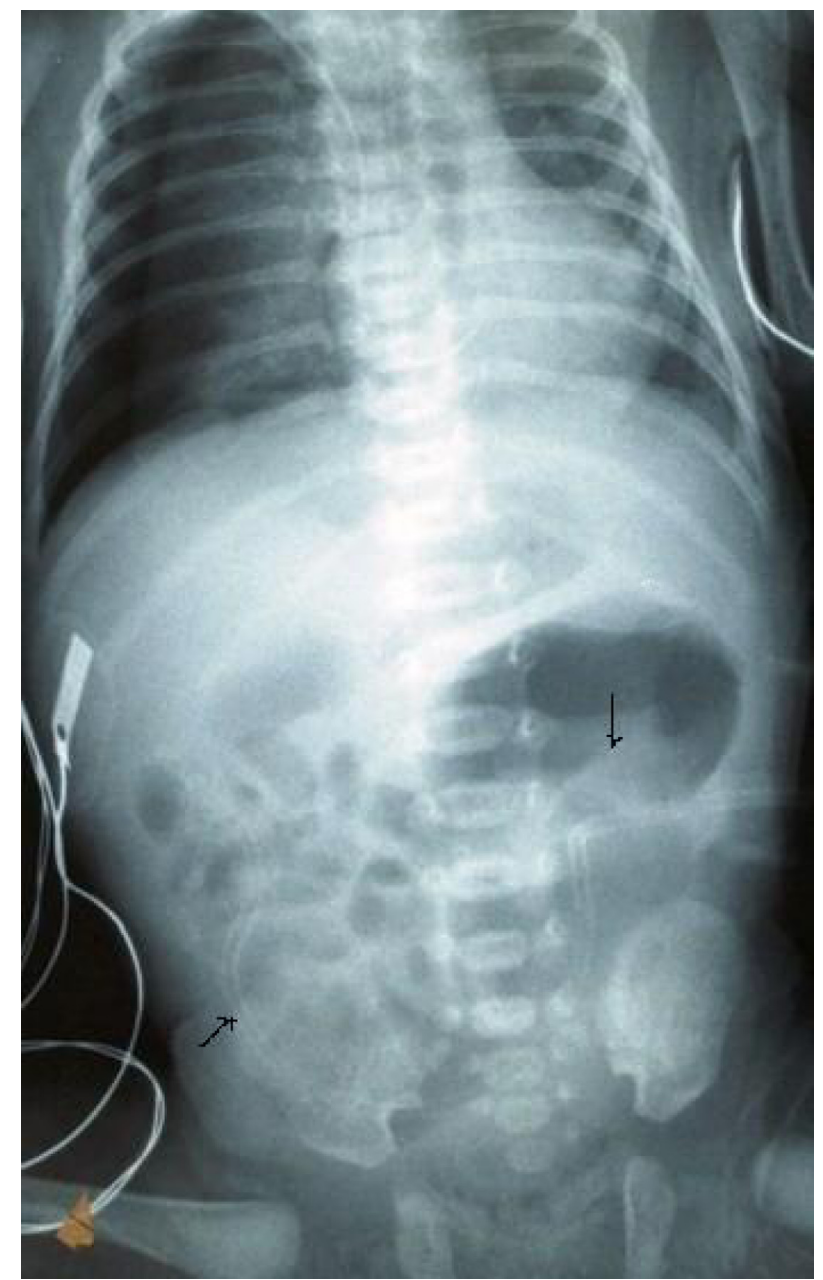

Figure 4 Abdominal X-ray 5 days after the operation shows air shadow in the apple peel intestinal segment, twin I. The Foley catheter is not seen as it is radio transparent.

$15 \mathrm{~mL}$ every 3 hours by day 34. Full oral feeding started on day 40 and adjunct parenteral nutrition was discontinued (total parenteral alimentation started on day 3 and decreased progressively according to their oral-feeding tolerance). Transanastomotic stent was removed on day 22 postoperative, but the Foley catheter was kept for 3 months. During this period, meals were divided and given frequently.

In both cases, the same surgical technique was applied with full satisfaction. In follow-up for more than 1 year, both cases reached normal developmental milestones.

\section{Discussion}

Intestinal atresia is the most common cause of neonatal intestinal obstruction, occurring at a rate of 2.9 per 10,000 births (including stillbirths). Sex distribution is almost equal. Apple peel atresia, which represents $<10 \%$ of all types of intestinal atresia, is defined as complete separation of blind ends with an extensive mesenteric defect where the distal small intestine coils around the axis of blood supply, acquiring its blood supply from a single ileocecal artery. ${ }^{1}$ In these types of congenital anomalies the overall bowel length is shorter than normal and usually accompanies extreme prematurity. Hereditary predisposition is also reported.

Apple peel atresia poses challenging problems particularly for reconstruction. The most recommended surgical repair for apple peel atresia consists of a proximal jejunostomy (usually with tapering or plication of the dilated segment to enhance its return to a normal caliber and active peristalsis) and ileostomy (Mikulicz exteriorization) or distal tube ileostomy at the level of distal blind-end loop. ${ }^{2-4}$ The latter is used later on for very progressive fluid administration before scheduling a definitive anastomosis. In this condition, definitive anastomosis is usually not possible before the age of 3-6 months. This method leads to high mortality due to the major complications of proximal jejunostomy in the neonatal period and the complications of long-term total parenteral alimentation. ${ }^{5}$ Previously, this method was applied without any incident in several cases of simple jejunal atresia and in many referred multioperated cases for different causes of proximal intestinal obstruction.

Primary anastomosis is protected by proximal stomy and disruption should not occur if anastomosis was correctly done. Decompression of the proximal loop is maintained as long as needed by the Foley catheter without any related complication; it does not interrupt the intestinal transit even when it is kept open permanently. Ten days after insertion, the Foley catheter can easily be replaced in case it falls out (like gastrostomy tube). The Foley catheter should not be removed before the proximal loop returns to its adequate caliber (no more dilatation on X-ray). Plication or tapering the proximal dilated loop is unnecessary as well as partial resection (except when dilated end-loop is in a cystic shape). Foley catheter can be opened to relieve any episodes of vomiting or abdominal distention.

The transanastomotic tube (stent) enables the content of the proximal intestinal loop to reach the distal loop progressively and does not prevent stricture at the site of the anastomosis. The stent can be removed a few days after the intestinal air shadow reaches the rectum and oral feeding can start to be tolerated. This small-sized transanastomotic tube should be inserted in conjunction with the Foley catheter rather than nasogastric - for better tolerance, and can be left in place for months without any related complication.

The most surprising issue in these case reports was that in spite of this typical aspect of apple peel atresia, and the complete disconnection from sympathetic and parasympathetic 
innervations, full early oral feeding was tolerated without any incident, except for mild chronic diarrhea with steatorrhea. There was no indication for intestinal contrast examination since the patients did not show any intestinal transit difficulties.

This method is recommended as first-line therapeutic approach for apple peel atresia as well as for different types of proximal jejunal atresia (with proximal dilated loop).

\section{Disclosure}

The author reports no conflicts of interest in this work.

\section{References}

1. Walker K, Badawi N, Hamid CH, et al. A population-based study of the outcome after small bowel atresia/stenosis in New South Wales and the Australian Capital Territory, Australia, 1992-2003. J Pediatr Surg. 2008;43(3):484-488.

2. Turnock RR, Brereton RJ, Spitz L, Kiely EM. Primary anastomosis in apple-peel bowel syndrome. J Pediatr Surg. 1991;26(6):718-720.

3. Harper L, Michel JL, de Napoli-Cocci S, et al. One-step management of apple-peel atresia. Acta Chir Belg. 2009;109(6):775-777.

4. Federici S, Domenichelli V, Antonellini C, Domini R. Multiple intestinal atresia with apple peel syndrome: successful treatment by five end-to-end anastomoses, jejunostomy, and transanastomotic silicon stent. J Pediatr Surg. 2003;38(8):1250-1252.

5. Festen S, Brevoord JC, Goldhoorn GA, et al. Excellent long-term outcome for survivors of apple peel atresia. J Pediatr Surg. 2002;37(1):61-65.
Open Access Surgery

\section{Publish your work in this journal}

Open Access Surgery is an international, peer-reviewed, open access journal that focuses on all aspects of surgical procedures and interventions. Patient care around the peri-operative period and patient outcomes post surgery are key topics. All grades of surgery from minor cosmetic interventions to major surgical procedures are covered. Novel techniques

\section{Dovepress}

and the utilization of new instruments and materials, including implants and prostheses that optimize outcomes constitute major areas of interest. The manuscript management system is completely online and includes a very quick and fair peer-review system. Visit http://www.dovepress.com/ testimonials.php to read real quotes from published authors. 